\title{
An Investigation of the Relationship between Organizational Structure, Tie Strength and Tacit Knowledge Sharing
}

\author{
Hoda Awada \\ The American University of Beirut
}

Received: 18 May 2019

Revised: 24 June 2019

Accepted : 26 June 2019

\begin{abstract}
Previous research firmly established the relation between tie strength and type of knowledge shared in a project or organization. However, the investigation into the effect organizational structure has on both tie strength and knowledge sharing is understudied. This paper investigates the impact of organizational structure on the formation of relationships and tacit knowledge sharing, through understanding networks and the role of tie strength. With the use of a single case study, I collected data and analysed results based on interviews and questionnaires with actors in an advertising and communication firm in Beirut, Lebanon. The latter occurred by studying the organization's network across boundaries, through examining interpersonal relationships spanning both intra-organizational and inter-organizational networks. Findings proved a relation between tie strength and knowledge sharing, and further signified the importance knowledge transfer has on organizational effectiveness with emphasis on knowledge management. Additionally, contributions were made regarding network definitions and the importance of considering organizational structure in the analysis of a firm as it can impact the strength of a tie.
\end{abstract}

Keywords:

Organizational Structure, Tie Strength, Knowledge Sharing, Networks, Organizational Performance

\section{Introduction}

Organizational structure includes a firm's hierarchy, decision-making centre, task division and relationship formation (Ahmady, Mehrpour and Nikooravesh., 2016). Its interactions can be studied in terms of network relationships in the field of social network analysis to explore the flow of resources such as knowledge between actors; including individuals or firms (Lei and Xin, 2011). The importance of knowledge, how it is stored and shared in an organization has also become an important topic of exploration, as researchers have uncovered the influence knowledge management has on business performance (Miraglia and Wei, 2017). The latter helps individuals learn from previous mistakes and improve efficiency (Zheng, Yang and Mclean, 2010). To investigate the above, the 
structure of relationships in terms of tie strength must be examined as it creates the environment for the flow of the resource. However, how these relationships form in the first place is contingent upon the organization's overall structure (Mahmoudsalehi, Moradkhannejad and Safari, 2012). The interdependence among organizational structure, tie strength and knowledge sharing is apparent, yet, has not been particularly studied in literature. Thus, the aim of this paper is to study the effect of interpersonal relations in both internal and external networks to that of knowledge sharing while considering organizational structure.

\section{Theoretical Framework \\ Organizational Structure}

Organizational structure can be defined as the method in which work is divided, activities are executed and control is used through authority relationships; for the management of processes and achievement of goals (Ahmady, Mehrpour, and Nikooravesh, 2016). It includes internal relationships, information and communication flows, and how units are interrelated (Mintzberg, 1979). Structure encompasses linking mechanisms between roles and how they are managed, becoming an essential marker for understanding humans in projects and organizations (Handy, 1993). Recent research describes it as a complex social phenomenon used to respond to tasks, including individuals and their actions (Pryke, 2017). Social structure is evaluated on the factors of: formulization, centralization and complexity (Van De Ven 1979). Formulization depicts how rules and policies control relations. Centralization illustrates the power top management has on decision making. Finally, complexity refers to the number of interrelated factors that are involved in a relation, such as people or resources. In this respect structure can be seen as a medium where social interactions take place and relationships are established (Van De Ven 1976). Consequently, studies have begun including the role of structure when evaluating relations (Ahmady, Mehrpour and Nikooravesh 2016). The organization's structure, whether hierarchal or flat, can inhibit or facilitate the flow of knowledge (Rishipal, 2014). Hierarchies are tall with power concentrated at the top, causing knowledge sharing problems between units such as: lack of willingness to share, fear of speaking up and less saliency between layers (Handy 1993). Conversely, flat organizations are decentralized, allowing for more integration and information sharing. The latter has been revealed in research investigation relationship (Reitzig and Maciejovsky, 2015; Mahmoudsalehi, Moradkhannejad, and Safari, 2012; Willem and Buelens, 2009).

\section{Forming Networks}

Organizations are social in nature, composed of interrelated divisions that have complex linkages which depend on the flow of information and decisions (Mintzberg, 1979). These links are a result of individuals establishing countless relations, forming networks composed of actors or nodes to acquire and share project information (Pryke, 2017; Borgatti and Halgin, 2011). The investigation of relationships reveals to be deeper than a social interaction and can be explored by networks. Links are classified as 'pipes' where resources can flow and help explain how knowledge moves within a network. According to network theory, ties connect nodes and can be categorized as strong or weak. Studies have found that strong ties resemble a solid and close relation with large amounts of communication. They include trust and commitment which are time dependent and costly to establish. Contrarily, weak ties depict distant relations (Borgatti and Halgin, 2011; Levin and Cross, 2004). Granovetter (1977) additionally acknowledges the existence of absent ties, considering strong and weak ties as present. This signifies not only strength as an important factor but their existence as fundamental. Literature suggests that strong ties transfer large amount of knowledge, yet lead to redundancy, while weak ties, if used, generate new and innovative knowledge. Both ties allow 
transfers however, their strength determines the type of knowledge they hold (Borgatti and Halgin, 2011; Phelps, Heidl and Wadhwa, 2012), and is essential for understanding the performance of networks in an organizational setting (Pryke, 2017). Subsequently, I chose to investigate the strength of the relationship since it has the ability to reveal greater insight into the type of knowledge shared.

\section{Knowledge and Its Use in an Organizational Setting}

For firms to gain competitive advantage, their capability to create and use knowledge is fundamental. Consequently, assessing knowledge flows between actors is vital (Zheng, Yang, and Mclean 2010). Knowledge can be defined as: the result of a certain activity and a medium to perform that activity (Mueller, 2015). It is categorized as: documented, structured and easy to share: 'explicit'; or subjective skilful and hard to articulate: 'tacit'. Explicit knowledge can be recorded and easily applied, providing basic procedural information; while tacit knowledge is subjective and hard to formally share as it holds individual experiences (Daniel, McCalla and Schwier, 2008). It is argued that both types of knowledge need to be shared for innovation. However, sharing knowledge to the right person at the right time is integral. Knowledge management is a process that facilitates how activities occur by generating, sharing and utilizing knowledge within an organization (Mahmoudsalehi, Moradkhannejad and Safari, 2012). Knowledge has revealed to be found in its individuals, organizational tools and within employee tasks, making it hard to measure (Dhanaraj, Lyles, Steensma and Tihanyi, 2004). Furthermore, the ability to acquire knowledge depends on the relation an actor has with a knowledge source (Levin and Cross, 2004). For the transfer of knowledge a network of interrelated and coordinated relations must be established, especially if the shared resource has a tacit component (Szulanski,1996; Daniel, McCalla and Schwier, 2008). Here, the role of ties is critical since strong ties have proven to transfer tacit knowledge as they involve familiar relationships. Weak ties, despite allowing for less transfer, offer new innovative knowledge, contributing to its creation (Phelps, Heidl, and Wadhwa, 2012). Additionally, weak ties impact how knowledge is diffused. They have proven to spread knowledge to a wider network of actors since they include bridges which are responsible for connecting two unrelated actors; increasing the number of overall ties created. These bridges can exist within an organization as well as between people in different organizations, and without them networks would be fragmented (Granovetter, 1977; Huang et al, 2016). In an organizational setting, knowledge is the pillar to its performance and viability, making both its availability and management critical.

\section{The Importance of Knowledge Management and the Role of Organizational Structure}

Knowledge management is dependent on the structure of both relations formed and the organization as a whole (Mahmoudsalehi, Moradkhannejad, and Safari, 2012). Structure affects processes by shaping patterns, improving communication and allowing for innovation. It helps establish organizational knowledge, coordinate processes and work activities (Zheng, Yang, and Mclean 2010). It is argued that an organization's structure influences knowledge sharing since it creates the place for the exchange (Willem and Buelens, 2009; Mueller, 2015). Conclusively, social interactions (network and relationship formation) play a mediating role between organizational structure and knowledge (Chen and Huang, 2007). Recent research has uncovered the positive relation knowledge management has on organizational effectiveness, ensuring lessons learned and minimizing repeated mistakes (Zheng, Yang and Mclean, 2010; Miraglia and Wei, 2017). For the above to occur, organizations need access to knowledge from external relations formed with individuals in other firms, in addition to creating relations between individuals within the firm (Tsai, 2001). 


\section{Interpersonal Relations and Knowledge Transfer in Intra-Organizational and Inter- Organizational Networks}

In order to adequately assess the impact of interpersonal relations on a firm or project performance, both intra-organizational and inter-organizational relations should be considered (Pryke, 2017). Intraorganizational networks are composed of both formal and informal relations. Formal relations show lines of authority, whereas informal relations are based on personal interactions and interpersonal ties between individuals (Witteck, 2014). Interpersonal relations are important for the transfer of knowledge, since, when individuals acquire knowledge, establishing links with other actors is necessary for its sharing, increasing understanding and awareness (Yang and Maxwell, 2011). Strong ties prove significant levels of socialization and knowledge transfer, increasing the know-how of where to search for knowledge; yet comes at a cost of less diversity (Phelps, Heidl and Wadhwa, 2012). Interpersonal linkages are vital for knowledge utilization, ensuring effective diffusion and application, particularly in less centralized and formulized organizations (Chen, and Huang, 2007). Networks should be studied from a holistic approach since knowledge shared in interpersonal relations between individuals in different firms can be accessed and diffused properly within a firm (Pryke, 2017). Actors create these ties, representing a link between the external and internal environment, acting as bridges. All bridges are considered weak ties, providing access from a variety of resources (Granovetter, 1977). Local create shorter paths between non-connected actors. Here, actors with many weak ties can quickly share more novel knowledge. This is particularly interesting when examining the network across internal boundaries as bridges between internal and external actors can be investigated. Creating inter-organizational relations helps knowledge spill overs (Borgatti, and Halgin, 2011). However, Bocquet and Mothe (2010) argue that this is only effective if properly communicated. Governance ensures that knowledge is usable within organizations by gathering external sources, classifying them and delivering the relevant resources. The nature of the relationship and how it is managed depicts whether the information individuals receive is repetitive explicit or innovative implicit knowledge. If this ability is limited, development and problem resolution will be compromised, hindering effectiveness.

\section{Hypothesis Development}

Firms engage in social interactions and, based on the above, the external setting creates the resources that are to be used within the internal environment (Yang, and Maxwell, 2011). To successfully study inter-organizational knowledge transfer, identifying whether the information is acquired internally in an organization is fundamental (Yang and Maxwell, 2011). Subsequently, internal and external aspects should be considered in conjunction when examining relationship building (Palmer, 1996). Phelps, Heidl and Wadhwa (2012) offer a literature on various studies regarding networks, knowledge transfer and utilization, categorizing the studies into those that examined: interpersonal, intraorganizational and inter-organizations. Based on their results, and in addition to the literature included in this paper, minimal research has delved into networks as a combination of interpersonal relations in both intra-organizational and inter-organizational levels particularly while studying knowledge sharing. Subsequently, the posed research question is: 'How does the strength of interpersonal ties on an intra-organizational and inter-firm level affect the ability to transfer and utilize tacit knowledge effectively within an organization'. Accordingly,

Hyp 1: The extent of tacit knowledge transfer increases in organizations with strong interpersonal ties Hyp 2: The extent of tacit knowledge transfer increases between actors in different firms with strong interpersonal ties

Hyp 3: The role of bridges helps increase the extent of tacit knowledge transfer 


\section{Research Design and Context}

Using a single case study enabled an extensive investigation into an organization, allowing for the understanding of a particular business (Quinlan and Zikmund 2015). A communication agency in Beirut, Lebanon that specializes in marketing and advertising strategies was used. Their work depends on combining creative, digital and public relations departments to cater for distinct clients. As advertising lies within a creative industry, innovative information is key; making tacit knowledge a fundamental factor for effective production.

\section{Methods and Measures}

Social network analysis (SNA) technique was used for data collection, a beneficial approach when trying to understand how links are formed and resources are exchanged (Daniel, McCalla and Schwier, 2008). Holding a statistical nature, SNA faces criticism including the inability to prove causality and having a high level of complexity. Pryke (2017), counter argues criticism, highlighting the importance of combining both SNA and qualitative information to effectively study human interactions. Accordingly, using a mixed approach, SNA includes gathering information from questionnaires and interviews to gain in-depth knowledge regarding relationships created (Serrat, 2017). Here, forty minute face-to-face interviews based on a questionnaire were carried out, followed by an open discussion to reveal further insight for analysis. Interviews were initiated by general questions (Table 4). Following, the questionnaire on tie strength and knowledge sharing was completed. Tie strength is the independent variable represented mathematically and approximated by the following formula of weighted averages: $T S{ }^{A-B}=f^{A-B} x^{A-B}$. It aims to study tie strength between nodes A and B, and suitable for this research (Pryke 2017). "f" represents the frequency of communication, and "i" the importance of the resource in the mind of the receiver. Questions are based on a questionnaire by Pryke (2012) that explores tie strength and knowledge flows but were adjusted to fit the case at hand. Participants were first given a Likert scale from 1-9 representing the frequency of interaction: 1 being every couple of months and 9 being several times a day. Second, the nature of the information being communicated was assessed and classified as: $\mathrm{A}=$ instruction; $\mathrm{B}=$ advice; $\mathrm{C}=$ information; $\mathrm{D}=$ discussion. Thirdly, the importance of the information they receive was ranked using a Likert scale from 1-9; 1 being irrelevant/not applicable and 9 being extensively important. For knowledge sharing, Willem and Buelens (2009) used the complexity of sharing knowledge as an indicator of its tacit nature. They relied on the use of proxies to understand how knowledge was shared such as the amount of time taken to explain an idea. To quantify the latter, individuals rate each response following a Likert scale ranging from 1 to 5,1 being minimal amount and 5 a substantial one. Finally, the third Hypothesis has been established to study how tacit knowledge is shared between actors through identifying bridges. This is essential to investigate since some individuals may be receiving information through indirect ties. Determined in the follow up questions, this Hypothesis will support Hypotheses 1 and 2, giving the study greater validity in terms of evaluating the extent of tacit knowledge sharing and the importance to tie strength. When assessing intra-organizational ties, a network of connections among all participants will be mapped, depicting the strength of relations within the chosen boundaries. Whereas, on the inter-organizational level, the relations will be explored strictly by interviewing individuals in the case study firm. Therefore, the network data in this research is not triangulated due to the limitation of time and access to participants. 


\section{Research Findings Quantitative Findings}

To analyse the data, a Pearson correlation coefficient (r) was used as it is suitable when studying the above variables in network analysis; particularly when tie strength is given a value, rather than merely stating the presence of a relationship (Hanneman and Riddle, 2005). There are multiple data sets with each actor holding their own results relating to tie strength and type of knowledge shared. First, $r$ was calculated for each actor. Second, a Fisher $\mathrm{z}$ transformation was performed followed by averaging the $z$ ' of all actors and transforming it back to $r$ which represents the average correlation between tie strength and knowledge sharing. Though z' can be considered as a suitable indication of a correlation, the final conversion to $r$ was computed to overcome bias and overestimations; since the standard error of average $r$ is known to be smaller than that of average $z$. This method is proven to be useful for small sample sizes such as the case at hand (Silver and Dunlap, 1987). Following, results from the questionnaires were converted into a socio-matrix and then mapped into a sociogram to help visualize the network.

Hypotheses 1 and 2: Results in Table 1 show the overall $\mathrm{r}$ for internal and external actors. There is a positive correlation between tacit knowledge sharing and tie strength in both internal and external settings. For Hypothesis $1, \mathrm{r}=0.44$, representing a moderate positive relation. Hypothesis 2 was confirmed with a correlation of $\mathrm{r}=0.68$, signifying a strong positive correlation.

Hypothesis 3: No results were obtained since no bridge was found in the organization.

Table 1. Averages of individual $r$ and $z$ values, overall $r$ for internal actors, overall $r$ for external actors

\begin{tabular}{ll}
\hline Average of individual $r$ (internal actors) & 0.37 \\
\hline Average individual $\mathrm{z}$ (internal actors) & 0.47 \\
\hline Transformation of $\mathrm{z}$ to $\mathrm{r}$ (internal actors) & 0.44 \\
\hline External $\mathrm{r}$ & 0.65 \\
\hline
\end{tabular}

Table 2. Average percentage of strong ties and tacit knowledge

\begin{tabular}{lll}
\hline Network & Average percentage of strong ties & Average percentage of tacit knowledge \\
\hline Internal & 43.18 & 68.99 \\
\hline External & 43.86 & 71.43 \\
\hline
\end{tabular}

Results in Table 2 were obtained from the questionnaire answers (based on the Likert scales). When tie strength $=>40.5$ it was considered strong, similarly when knowledge $>=3$, it was considered tacit and non-tacit if $K<2$. All relations were further depicted in the below sociogram (Figure 1), where each coloured line signifies the combination tie strength and type of knowledge. Relations were calculated in both directions and were not averaged and rather represented individually, overcoming simplification and allowing additional insight into the relation (Pryke, 2017). 


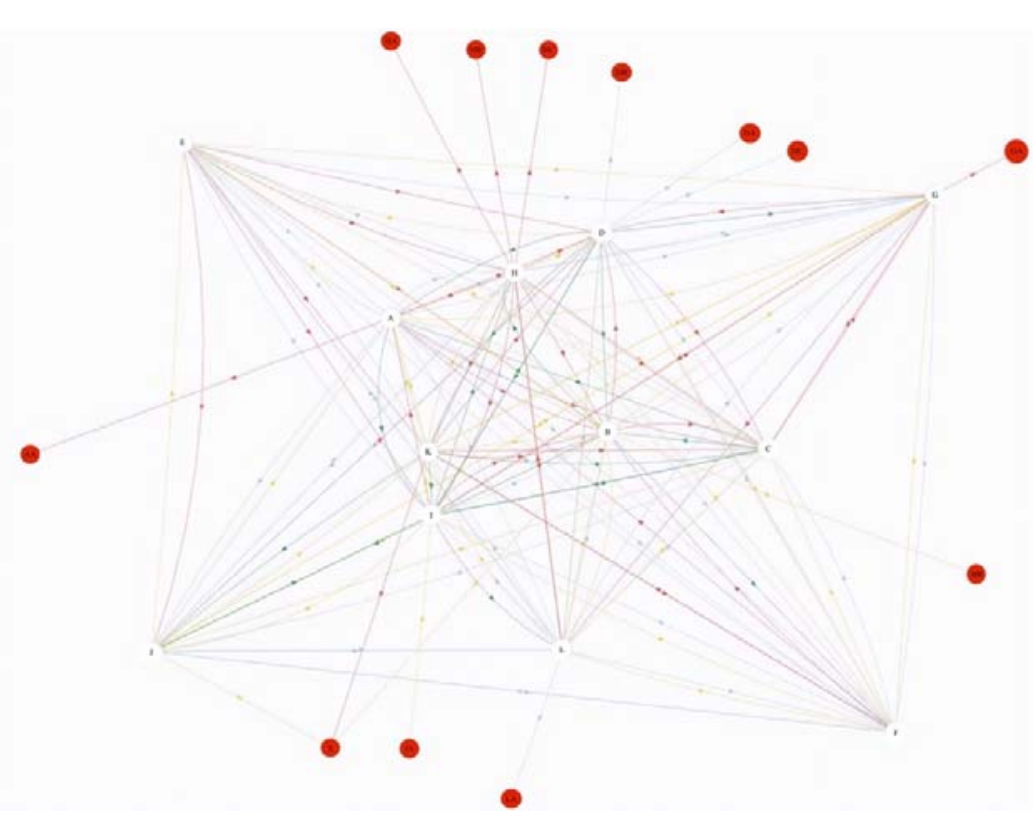

Figure 1. Sociogram

Table 3. Sociogram Legend

\begin{tabular}{llll}
\hline $\mathrm{ST}+\mathrm{TK}$ & Red & WT+TK & Blue \\
\hline ST+NTK & Green & WT+NTK & Yellow \\
\hline
\end{tabular}

\section{Qualitative Findings}

Table 4. Qualitative findings from general and follow up questions

\section{Organization}

What type of structure would you say your organization has?

How would you describe the culture of your organization?

What would you say your organization has as a competitive advantage over other communication agencies?

\section{Factors affecting relation formation}

Would you say trust affects the type of relationship you have with those around you?

Do you think the organizations structure impacts the type of relationships you from?

In terms of creating these relationships, would you consider time as an important factor?

Do the relationships you hold impact the ability to perform your work?

\section{Factors affecting knowledge transfer}

Do you think the organizations structure impacts the ability to share knowledge with others?

\section{Tacit knowledge}

Does the type of relationship you have with individuals around you affect whether you share knowledge with them or not?
$75 \%$ said that the structure is more of a flat organization, but hierarchy in terms of top management and decision making exists

$75 \%$ of respondents stated that the environment is: very friendly having an open culture, all of which makes them feel valued

$58 \%$ mentioned their unique structure and knowledgeable human capital

$100 \%$ of the participants responded that trust heavily impacts relationship formation and affects their ability to work in terms of depending on one another

$100 \%$ agreed that their open space and their flat structure allows relationship formation

$75 \%$ responded that time was not an apparent factor. $100 \%$ said it depends on the person the relationship is being formed with

$83 \%$ confirmed that the relationships they hold impacts their ability to work

$100 \%$ stated that the organizations structure allows for sharing due to their open space and ability to contact anyone

$83 \%$ stated that they share knowledge regardless of the strength of relationship and for the purpose of improving performance 


\begin{tabular}{|c|c|}
\hline $\begin{array}{l}\text { Do you think you have access to knowledge in the firm? If } \\
\text { so, where do you find this knowledge? }\end{array}$ & $\begin{array}{l}92 \% \text { expressed their access to knowledge within the firm, with } \\
\text { particular reference to the relations they have. }\end{array}$ \\
\hline $\begin{array}{l}\text { What type of knowledge does your work depend on? Tacit } \\
\text { or non- tacit? }\end{array}$ & $58 \%$ work depends on tacit knowledge \\
\hline $\begin{array}{l}\text { In terms of improving project performance which source of } \\
\text { knowledge received has additional value? that obtained } \\
\text { internally or externally? }\end{array}$ & $\begin{array}{l}37 \% \text { stated that internal knowledge adds more value, } 37 \% \text { said } \\
\text { it was that of external and the remaining } 26 \% \text { agreed that both } \\
\text { internal and external knowledge add value to their work. }\end{array}$ \\
\hline $\begin{array}{l}\text { Has knowledge sharing allowed you to improve project } \\
\text { performance in terms of client satisfaction and value } \\
\text { enhancement? }\end{array}$ & $\begin{array}{l}100 \% \text { confirmed that knowledge sharing has improved project } \\
\text { performance }\end{array}$ \\
\hline $\begin{array}{l}\text { Has knowledge sharing allowed you to learn from previous } \\
\text { mistakes or avoid their repetition? }\end{array}$ & $\begin{array}{l}92 \% \text { revealed that their ability to share knowledge has allowed } \\
\text { for lessons learned and decreased in repeated mistakes }\end{array}$ \\
\hline
\end{tabular}

The above qualitative results provided awareness on the nature of the environment such as: the culture, organizational structure, performance, relationship formation and knowledge flow. The structure revealed to be in between flat and hierarchy where all interviewees articulated the ability to have direct contact with top management. Relationships appeared to be strong and close, with participants conveying the capability to create and maintain bonds.

\section{Discussion}

\section{Organizational Structure}

The firm's structure appeared to impact relationship formation. First, having open offices allows direct access to one another. Second, on an operational level, actors work in teams of three composed of one creative, digital and client servicing individual that caters to the same clients, overcoming fragmentation in their work. This allows for good work relations, facilitating interaction and ensuring proper involvement of all parties- Actor C. Additionally, they merged different features of advertising under one roof, shown as separate entities on the edges in the sociogram (Actors I, J, G, H). They are considered independent units in terms of their execution, yet heavily rely on internal actors for their work. In terms of social structure, the organization was found to be complex, non-formulized and with some centralization in top management. Participants revealed that their structure allows them to express and communicate ideas, escaping information sharing problems (Reitzig and Maciejovsky 2015). Despite the above, it was mentioned that with less formalization, chaos arises. While not affecting relationship formation, it hinders effectiveness, as actors overlook protocol and waste time socializing.

\section{Tie Strength}

The correlation of tacit knowledge was found to be moderate on the intra-organizational level. This could have resulted from the chosen sample. Having interviewed actors varying from those on an operational level to heads of departments and partners, resulted in lowering the overall correlation value with $\mathrm{r}=0.65$ (Table 1). External ties are dependent on trust resulting in long lasting relations. This was apparent when interviewees explained a high reliance on external parties to perform their work including the need for suppliers, information and establishing contacts.

\section{Knowledge}

As a result of their interaction, communication, informal relations, and information sharing, the organization's structure allows sufficient tacit knowledge sharing (Daniel, McCalla and Schwier, 2008). Interviewees articulated the close personal relationships they have with their colleagues which has instilled a non-dependent intention to share knowledge. Likewise, experts act as mentors enhancing individual learning (Yang and Maxwell, 2011). Actor L revealed that instant relationships 
are formed with members on a personal level allowing them to promptly help those around. Knowledge application was also based on how valid they perceive the knowledge the sender holds, depending on how relevant it is for the project itself and the person's know-how (Phelps, Heidl and Wadhwa, 2012). Furthermore, structure appears to be a fundamental factor for knowledge sharing and interpersonal relations allow for its application (Chen and Huang, 2007). Finally, the amount of tacit knowledge could have resulted from creativity and innovation which are valued and supported by their structure where team rotations occur frequently generating new ideas for the client.

\section{The Role of Tie Strength and Tacit Knowledge Sharing}

Interpersonal tie strength of both intra-organizational and inter-organizational levels appeared to be composed largely of weak ties that were tacit. Referring to table 2, the average percentage of internal strong ties was approximately $43.2 \%$, and that of external at $42.8 \%$. The corresponding averages of tacit knowledge were $68.9 \%$ and $71.4 \%$ respectively. Interestingly, the percentages of strong ties were also high, hence the notion of strength solely determining the type of knowledge is an exaggerated claim here. Hypotheses 1 and 2 support the idea that strong ties transfer tacit knowledge (Phelps, Heidl and Wadhwa, 2012). The notion of weak ties creating knowledge is also proven. Actor D states external individuals add the 'cherry on top' to the work they perform. However, these claims cannot be generalized. The relation between Actor $\mathrm{C}$ to $\mathrm{D}$ is $\mathrm{WT}+\mathrm{TK}$ but from Actor D to C is ST+TK (Figure 1 and Table 3). This shows that tacit knowledge is being shared regardless, giving the existence of ties' importance rather than their strength (Granovetter 1977). Although no bridge was identified internally, an intermediary between internal and external members was recognized. Actors $\mathrm{A}, \mathrm{B}$ and $\mathrm{K}$ (client servicing) who provide others with needed information and knowledge are considered bridges between the external actors and the creatives (internal). Actor B, which has a weak tie to Actor BA, makes them a possible bridge since without their role a project could not be fully executed. This weak tie confirms the importance of bridges in overcoming network fragmentation. Moreover, this could contribute to spreading knowledge to a wider network (Granovetter, 1977). In response to the research question proposed, the findings indicate that the tie strength affected how the firm transfers knowledge.

\section{Sociogram Analysis}

The actor's role seemed to play a significant part in the ties individuals have, apparent in the different types of identified relations. For example, internally, Actors A and B work across from one another allowing them to constantly share knowledge; accordingly, the relation from B-A is ST+TK and A-B is WT+TK (Table 3). Relationship strength appeared to be subjective, however the type of knowledge is tacit both ways. Conversely, Actor K sits far from Actor A and has a WT+NTK, justifying the influence an organization's structure has on tie strength. Actor H however, has ST $+\mathrm{TK}$ represented by red lines with Actors A, B, C, D who are responsible for the project and give Actor $\mathrm{H}$ the needed tasks to be executed. Here, a strong tie results from the frequency of interaction and tacit knowledge is a given since creativity is always present. Actor $\mathrm{H}$ however does not work with Actors E, F, G, making the relation is WT+TK. Having a present tie appears to have a greater impact than its strength since there are many factors to be considered such as the type of project or the project life cycle which was articulated by interviewees (Granovetter, 1977). Moreover, the use of the sociogram proved that the causal relation hypothesized between tie strength and type of knowledge transfer is positive, yet other external factors do play a role; ultimately displaying the complexity of the relation. 


\section{Organizational Performance and Knowledge Management}

Individuals have easy access to knowledge, yet, this occurs through direct contact and not through the use of a knowledge management system. With their structure and open access, such a system may be irrelevant. Nevertheless, the lack of record could hinder performance where multinational companies may poach their highly-skilled staff. Additionally, establishing a system ensures that proper information is provided to the right person at the right time (Mahmoudsalehi, Moradkhannejad and Safari, 2012). This will help improve effectiveness by enhancing problem-solving capabilities (Zheng, Yang and Mclean 2010). Instilling governance will also ensure that knowledge is not only shared but utilized. This is pivotal to help evaluate whether external expert knowledge is being diffused into the organization and can be investigated through bridges (Granovetter, 1977; Bocquet and Mothe, 2010).

\section{Conclusions and Recommendations}

What prompted this study was the question of how tie strength impacts the ability to transfer and utilize knowledge within a firm by taking a holistic approach. Our results proved structure to be a medium of exchange with an apparent impact on forming strong relationships through which resources such as knowledge can flow (Ahmady, Mehrpour and Nikooravesh, 2016). Weak ties proved to transfer innovative knowledge. The claim that strong ties depict more communication and knowledge than weak ties did not appear to be a general rule. The actors' frequency of interaction might differ between different project stages resulting in weak ties, yet are still close and based on trust. When studying ties from both directions, strength differed with perception playing a role, but the type of knowledge shared was consistent. Thus, literature stating that tacit knowledge transfer depends on tie strength may be lacking. This is supported by results showing that tacit knowledge was shared regardless of strength. Finally, the diffusion of knowledge is what enhances organizational performance which can be reinforced by knowledge management. Here diffusion took place in both weak and strong ties. The term of strength needs to be revisited since using 'weak' or 'strong' is looking at a network in a particular setting. This can be especially misleading in a project-based organization, with changing team members and relationships. There is a need to understand the context of the project in a structural analysis of an organization; which could have impacted Hypothesis 3 (Pryke, 2017). Due to direct connections, the role of bridges is immediately diminished. It is probable that if more individuals were interviewed a bridge could have been identified. Furthermore, tie strength is correlated to the type of knowledge shared, yet other factors come into consideration that make this theorization weak. Elements such as actor's role, level of dependence, time in project life cycle and organization's structure all impact the frequency of interaction. Accordingly, existence rather than strength is important. With regards to the type of knowledge shared, this was also highly contingent on the type of industry, level of informality and culture in the organization. Conclusively, organizational structure affects tie strength which then impacts knowledge sharing. Findings contribute to literature by assessing the interference organizational structure has on network abilities, with an emphasis on redefining strength and focusing on its presence.

\section{Limitations and Future Research}

The above conclusions are based on the particular firm studied and cannot be generalized. This research was conducted for an MSc dissertation, resulting in limitations from the use of a single case research method due to time constraints and limited access to participants. This hindered the ability to reach complete conclusive remarks which could have been made if: bridges were identified and additional internal and external actors were considered, allowing for triangulated data. Nevertheless, the definition of strength, significance of organizational structure in networks and its impact on 
knowledge sharing and utilization should be revisited. Future research should recognize findings from both internal and external settings without limiting the scope to project networks and factors such as tie strength. Investigating why these ties form in the first place and understanding the whole network will then define what 'weak' or 'strong' means in that particular setting, allowing for richer conclusions.

\section{References}

Ahmady, G. A., Mehrpour, M. and Nikooravesh, A.(2016).'Organizational Structure',Procedia-Social and Behavioural Sciences, pp.455-462.doi: 10.1016/j.sbspro.2016.09.057

Bocquet, R. and Mothe, C. (2010). 'Knowledge governance within clusters: the case of small firms', Knowledge Management Research \& Practice, 8(3), pp. 229-239. doi: 10.1057/kmrp.2010.14.

Borgatti, S. P. and Halgin, D. S. (2011) 'On Network Theory', Organization Science. INFORMS: Institute for Operations Research, 22(5), pp. 1168-1181. Available at: http://10.0.5.7/orsc.1100.0641.

Chen, C.-J. and Huang, J.-W. (2007) 'How organizational climate and structure affect knowledge management-The social interaction perspective', International Journal of Information Management, 27(2), pp. 104-118. doi: https://doi.org/10.1016/j.ijinfomgt.2006.11.001.

Daniel, B. K., McCalla, G. I. and Schwier, R. A. (2008) 'Social Network Analysis techniques: implications for information and knowledge sharing in virtual learning communities', International journal of advanced media and communication. Inderscience Publishers, 2(1), pp. 20-34.

Dhanaraj, C., Lyles, M.A., Steensma, H.K. and Tihanyi, L.(2004).' Managing tacit and explicit knowledge transfer studies, 35(5), pp.428-442.

Granovetter, M. S. (1977) 'The strength of weak ties’, in Social networks. Elsevier, pp. 347-367.

Handy, C. (1993) 'Understanding Organizations', Journal of Sport Management, 21(4th), p. 444pp. doi: 10.1007/BF03176434.

Hanneman,R.A.and Riddle,M.(2005).Introduction to social network methods.Riverside.

Huang, Y., Luo, Y., Liu, Y., and Yang, Q. (2016)." An Investigation of Interpersonal Ties in Interorganizational Exchanges in Emerging Markets: A Boundary-Spanning Perspective." Journal of Management, 42(6), pp1557-1587.

Lei, G. and Xin, G. (2011) 'Social network analysis on knowledge sharing of scientific groups', Journal of System and Management Sciences, 1(3), pp. 79-89.

Levin, D. Z. and Cross, R. (2004) 'The Strength of Weak Ties You Can Trust: The Mediating Role of Trust in Effective Knowledge Transfer', Management Science. INFORMS: Institute for Operations Research, 50(11), pp. 1477-1490. Available at: http://10.0.5.7/mnsc.1030.0136.

Mahmoudsalehi, M., Moradkhannejad, R. and Safari, K. (2012) 'How knowledge management is affected by organizational structure', The Learning Organization, 19(6), pp. 518-528. doi: 10.1108/09696471211266974.

Mintzberg, H. (1979). The structuring of organizations: a synthesis of the research. Theory of Management Policy Series.

Miraglia, S.,Wei, C. (2017) Organizational culture and knowledge transfer in project-based organizations: Theoretical insights from a Chinese construction firm. International Journal of Project Management, 35 (4) pp. 571-585

Mueller, J. (2015) 'Formal and informal practices of knowledge sharing between project teams and enacted cultural characteristics', Project Management Journal, 46(1), pp. 53-68. doi: 10.1002/pmj.21471.

Palmer, A. (1996) 'Linking external and internal relationship building in networks of public and private sector organizations: a case study', International Journal of Public Sector Management. Emerald, 9(3), pp. 51-60. doi: $10.1108 / 09513559610124487$.

Phelps, C., Heidl, R. and Wadhwa, A. (2012) 'Knowledge, Networks, and Knowledge Networks: A Review and Research Agenda', Journal of Management. SAGE Publications Inc, 38(4), pp. 1115-1166. doi: 10.1177/0149206311432640.

Pryke,S.(2017).Managing Networks in Project- Based Organizations.Wiley.

Quinlan,C. and Zikmund, W.G.,(2015).Business Research Methods, Cengage Learning EMEA.

Rai, R.K. (2011). Knowledge management and organizational culture: a theoretical integrative framework. Journal of Knowledge Management, 15(5), 779-801.

Reitzig, M. \& Maciejovsky, B., 2015. Corporate hierarchy and vertical information flow inside the firm-a behavioral view. Strategic Management Journal, 36(13), pp.1979-1999. 
Rishipal (2014).'Analytical Comparison of Flat and Vertical Organizational Structures'.European Journal of Business and Management, 36(6), pp.56-65.

Serrat, O. (2017) 'Social Network Analysis BT - Knowledge Solutions: Tools, Methods, and Approaches to Drive Organizational Performance', in Serrat, O. (ed.). Singapore: Springer Singapore, pp. 39-43. doi: 10.1007/978-981-100983-9 9.

Szulanski, G. (1996) 'Exploring internal stickiness: Impediments to the transfer of best practice within the firm', Strategic Management Journal, 17(S2), pp. 27-43. doi: 10.1002/smj.4250171105.

Tsai, W. (2001) 'Knowledge Transfer in Intraorganizational Networks: Effects of Network Position and Absorptive Capacity on Business Unit Innovation and Performance', The Academy of Management Journal. Academy of Management, 44(5), pp. 996-1004. doi: 10.2307/3069443.

Van de Ven, A. H. (1976) 'On the Nature, Formation, and Maintenance of Relations among Organizations', The Academy of Management Review. Academy of Management, 1(4), pp. 24-36. doi: 10.2307/257722.

Willem, A. and Buelens, M. (2009) 'Knowledge sharing in inter-unit cooperative episodes: The impact of organizational structure dimensions', International Journal of Information Management, 29(2), pp. 151-160. doi: https://doi.org/10.1016/j.ijinfomgt.2008.06.004.

Wittek, R. (2014) 'Intra-Organizational Networks BT - Encyclopedia of Social Network Analysis and Mining', in Alhajj, R. and Rokne, J. (eds). New York, NY: Springer New York, pp. 766-774. doi: 10.1007/978-1-4614-6170-8_370.

Yang, T.-M. and Maxwell, T. A. (2011) 'Information-sharing in public organizations: A literature review of interpersonal, intra-organizational and inter-organizational success factors', Government Information Quarterly, 28(2), pp. 164-175. doi: https://doi.org/10.1016/j.giq.2010.06.008.

Zheng, Yang and Mclean, 2010. Linking organizational culture, structure, strategy, and organizational effectiveness: Mediating role of knowledge management. Journal of Business Research, 63(7), pp.763-771. 